\title{
Aplicación práctica de la metodología de caracterización geológico-minera al yacimiento de granito "Rosa Porriño" (Galicia, España). Cartografía de calidades y estimación y distribución de reservas para la planificación de su explotación
}

\author{
Á. Ferrero Arias ${ }^{(1)}$, J.Taboada Castro ${ }^{(2,4)}$, C. Iglesias Comesaña ${ }^{(2,4)}$, J.M. Baltuille Martín ${ }^{(3,4)}$, E. Giráldez Pérez(2)
}

(1) Instituto Geológico y Minero de España (IGME). Unidad de Santiago de Compostela.

C/. Cardeal Payá, 18, 1ㅜ, 15703 Santiago de Compostela (A Coruña). afaafa62@gmail.com

(2) Dpto. de Ingeniería de los Recursos Naturales y Medio Ambiente, Univ. de Vigo, Campus Lagoas Marcosende, 36310 Vigo (Pontevedra). jtaboada@uvigo.es, carlaiglesias@uvigo.es, egiraldez@uvigo.es

(3) Instituto Geológico y Minero de España (IGME). Dpto. de Infraestructura Geocientífica y Servicios. Ríos Rosas, 23, 28003 Madrid. jm.baltuille@igme.es

(4) Red CONSTRUROCK (Red de la Piedra Natural y su relación con el Patrimonio Histórico-Monumental y la obra nueva, en el ámbito del Estado español). Ríos Rosas, 23, 28003 Madrid.

\section{RESUMEN}

El granito "Rosa Porriño", mundialmente conocido y con una experiencia industrial-comercial de más de 50 años, es objeto de estudio en el presente trabajo mediante la aplicación de técnicas geológico-mineras para el análisis de macizos rocosos de cara a su aprovechamiento comercial. Se han tomado como punto de partida los criterios generales de caracterización de los parámetros geológicos y mineros: litologías y su distribución, tipos y localización de la fracturación, calidad industrial de la roca y ubicación de volúmenes extraíbles, relaciones de antecedencia, posibilidades de ordenación y racionalización de la explotación, etc.

La información geológico-minera obtenida se expresó mediante una cartografía de calidades industriales que constituyó la base para la estimación de reservas y su distribución. La aplicación de técnicas geoestadísticas ha permitido delimitar en tres dimensiones y obtener los volúmenes existentes de calidades industriales, propuestas en la cartografía geológico-minera. Se establecieron las reservas para varias calidades que incluyen calidades de primera y de segunda para roca ornamental, con usos de alto valor añadido; una tercera calidad para utilizar como piedra de construcción, en piezas en general menores; y una cuarta calidad con destino a áridos. Se delimitaron también las zonas estériles.

La estimación de las reservas de dichas calidades y su distribución espacial dentro del yacimiento ha permitido establecer alternativas de racionalización de las numerosas unidades de explotación existentes. De este modo se logra una operación minera más eficaz y eficiente, con impactos ambientales más controlados y reducidos, y, en consecuencia, un aprovechamiento más sostenible del recurso minero.

Palabras clave: geoestadística, granito, modelado 3D, recurso minero, yacimiento.

\section{A practical application of the geological and mining characterization method to the "Rosa Porriño" deposit (Galicia, Spain). Quality cartography and estimation of the distribution of reserves for mining exploitation planning}

\author{
ABSTRACT
}

This paper presents a study of the "Rosa Porriño" granite, which is known worldwide and has been marketed for more than 50 years, through the application of geologic-mining techniques. These techniques are wideIy used for analysing rock masses with the aim of commercially exploiting them. The basis is the general criteria for the characterization of geological and mining parameters: the lithologies present and their 
Ferrero Arias, A., et al., 2017. Aplicación práctica de la metodología de caracterización... Boletín Geológico y Minero, 128 (2): $451-466$

distribution, types and location of fractures, the industrial quality of the rock and the location of the exploitable rock volumes, previous activity, planning possibilities and rationalization of the exploitation, to name a few.

This geological and mining information was used to make a map of the industrial qualities, forming the basis for the estimation of reserves and their distribution within the deposit. The application of geostatistical techniques allowed the definition of the three dimensions and thus the existing volumes corresponding to each industrial quality (defined in the geological-mining cartography). Four different qualities were defined and their volumes estimated, namely first and second qualities for granite suitable for its use as ornamental stone or other applications of high added value; third quality for the granite suitable for its use as a construction material (generally of smaller volume than the previous qualities) and the fourth quality for the granite for aggregates. The overburden zones were determined as well.

Once the reserves of the qualities and their spatial distribution within the deposit are known, different alternatives for a rational exploitation can be considered for the existing operating units. This modelling enables a more effective and efficient mining operation, with controlled, lower environmental impact and thus with a more sustainable exploitation of the mining resources.

Keywords: 3D modeling, deposit, geostatistics, granite, mining resource.

\section{ABRIDGED ENGLISH VERSION}

\section{Introduction and Methods}

Granite has been used since ancient times in Galicia (NW Spain) for a wide number of applications such as megaliths, rock art, funerary structures, civil and religious monuments, as well as buildings. The locality of $O$ Porriño, located in the province of Pontevedra, is one of the main production centres of granite for ornamental use in the world.

Since being set up in March 2010, the Galician Granite Cluster has become the key association in this industrial sector, being considered as a strategic cluster for Galicia by the Xunta de Galicia in the "Strategic Plan Galicia 2010-2014. 2020 Horizon".

The granite industry is essential in Galicia due both to its production and turnover, being the leading autonomous community in granite production in Spain (more than half of the granite produced in the whole country is extracted in Galicia, with the majority being transformed in local workshops equipped with modern machinery). According to 2014 data (MINETUR), there are 94 active granite exploitations in Galicia (extracting granite for ornamental use in 53 of them), with a total of 798 workers, producing more than 5 million tonnes and valued at more than 47 million euros.

One of the main granite deposits is "Rosa Porriño," located in the city of the same name, and is used for ornamental use, for buildings and also as an aggregate, especially for concrete production. It has been studied as part of the exploitation project of concession "Benedicta" ( $\left.n^{\circ} 1.544\right)$, with the following objectives:

- General objective: obtaining the geological and mining data for the evaluation of the exploitable granite resources.

- Specific objectives:

- Detailed geological and mining mapping (E 1:3,500) of the area and its surroundings according to the different industrial qualities.

- Geological and mining mapping (E 1:10,000) of the whole concession of exploitation.

- 3D model of the exploited area, incorporating a map of the industrial qualities and other geological and mining information.

- Calculation of the reserves of the deposit by means of geo-statistics.

- Corresponding reports of the study.

The area of the exploitation, shown in Figure 1, occupies about $2 \mathrm{~km}^{2}(2 \mathrm{~km}$ long in NNW-SSE direction and $1 \mathrm{~km}$ wide). The aforementioned figure shows the different quarries existing in the "Benedicta" concession. Geologically speaking, the deposit is located in the granitic massif of O Porriño (Fig. 2), which is $25 \mathrm{~km}$ long in the N-S direction with widths ranging from $10 \mathrm{~km}$ to $15 \mathrm{~km}$. The O Porriño massif is part of GaliciaTras-os-Montes Zone, represented in this area by schist and gneiss materials of the Malpica-Tui Unit.

In order to achieve the specific 1 and 2 objectives, the general methodology for the study of granitic massifs for their use as ornamental stone (Muñoz de la Nava et al., 1989) was followed, adapting it to the conditions of the studied area, which had already been partly exploited. Thus, taking into account the expertise after many years of extraction and commercialization of the Rosa Porriño granite, the faces were systematically observed with the aim of obtaining a detailed cartography of the industrial qualities present in the quarry. 
Ferrero Arias, A., et al., 2017. Aplicación práctica de la metodología de caracterización... Boletín Geológico y Minero, 128 (2): $451-466$

Firstly, existing documents about the deposit were analysed, and the geologic and mining mapping (E 1:3,500) of the exploitation area was elaborated. In order to determine the homogeneous domains of the deposit in terms of quality, a number of basic parameters were considered, with fracturation being one of the most important ones due to its influence on the final use of the rock. With respect to quality, this was assigned according to the expertise of the miners. These data were collected systematically and represented in the aerial photograph E 1:5,000, as well as the information related to the vertical structure of the deposit, which was distributed in 30 observation stations. To complete the knowledge of the deposit, 5 drillings were made and their core samples studied and represented in columns E 1:100.

A comprehensive analysis of fracturation was carried out, measuring the direction, dip, distance between fractures and type of filler of the fractures in 8 transversal profiles, distinguishing between faults and fissures. Special attention was paid to the study of the "andar" plane, essential for the subsequent cutting in the workshop.

Photo-interpretation work, reconnaissance fieldwork and petrological studies were also performed in order to establish the different granitic facies.

Finally, all these data were included in a database to facilitate their analysis, and the cartography of industrial qualities was implemented with GIS methods.

The 3D model of the deposit was constructed using the distribution of qualities obtained by means of geostatistics, applying the fuzzy kriging method, which is especially appropriate when the degree of uncertainty is considerable (since the boundaries between the different commercial qualities are not clear, uncertainty is inherent to this work). For this purpose, 1,000 $\mathrm{m}^{3}$ blocks were defined, and the following qualities were considered: first quality is assigned to those rock masses whose ornamental quality is optimal and from which 6-12 $\mathrm{m}^{3}$ blocks can be obtained; second quality is for those volumes of good quality and from which up-to$6 \mathrm{~m}^{3}$ blocks can be obtained; third quality for rock masses from which semi-blocks of no more than $4 \mathrm{~m}^{3} \mathrm{can}$ be obtained; and fourth quality is for granite only valid for aggregates due to intense fracturation.

The deposit was divided into 1,000 $\mathrm{m}^{3}$ primary blocks, and fuzzy kriging was used to assign the qualities present in each block, bearing in mind that several qualities might be found in the same block. Figure 3 shows the triangular function used for the estimation of the qualities of the blocks of the whole deposit.

\section{Results and discussion}

The research performed has lead to a more comprehensive knowledge of the O Porriño massif, particularly the Rosa Porriño granite located in the "Benedicta" concession. According to the facies observed in the deposit, three commercial varieties have been identified: "Gris Mondariz" granite in the eastern part, "Porfidic Rosa Porriño" granite in the central part, and "Rosa Porriño" granite in the western part (Fig. 4).

With respect to the fracturation, the main planes are sub-horizontal: one of them is shown in Figure 5, with the space between fractures being more than $10 \mathrm{~m}$, whilst the other one (Fig. 6) is constituted by faults and fissures and presents shorter distances $(<2 m)$. This natural fracturation involves the need for artificial cutting for the extraction of the granite blocks. Regarding the main fault planes, they are defined by two conjugate fault planes in NNE-SSW and NNW-SSE direction, their dip ranging from $70^{\circ}$ to $90^{\circ}$.

Figure 7 shows the resulting homogeneous domains of commercial qualities for the ulterior geo-statistical treatment, whilst Figure 8 shows the simplified geological mapping of the exploitation area.

After the estimation of the distribution of qualities by means of the fuzzy kriging technique, the following results were obtained (Fig. 9): $13.89 \%$ corresponds to first quality, $15.96 \%$ to second quality, $21.59 \%$ to third quality and the remainder $48.56 \%$ to fourth quality. The results are included in the 3D model by means of a database that can be consulted through SQL queries.

The application of these geostatistical techniques to the Rosa Porriño deposit represents an advance on the existing techniques, since it provides more realistic estimations of the reserves based on the exploitation method, the anisotropy of the deposit and the fuzzy boundaries between the defined commercial qualities.

\section{Introducción}

Galicia ha sido desde tiempo inmemorial tierra de canteros ("canteiros"), utilizándose la roca granítica, que aflora extensamente en su territorio, en todos los usos constructivos, desde los más humildes y anti- guos (megalitos, soporte de arte rupestre, estructuras funerarias, cierre de fincas, postes...) a los más nobles y hasta la actualidad (monumentos tanto civiles como religiosos, construcciones y edificios tradicionales a contemporáneos).

Desde principios del siglo $\mathrm{XX}$ hasta ahora no ha 
dejado de ganar importancia el sector del granito en Galicia, en especial en la provincia de Pontevedra, concretamente en O Porriño, como roca ornamental, llegando a ser uno de los principales focos industriales de este material no solo en España sino también en el mundo.

En marzo de 2010 nace el Clúster del Granito de Galicia e integra toda la cadena de valor del granito, pasando a ser la Asociación de referencia de esta industria e interlocutor único del sector. La Xunta de Galicia lo considera como clúster estratégico para Galicia en el "Plan Estratégico Galicia 2010-2014 Horizonte 2020". Lo integran el Centro Tecnológico del Granito y las asociaciones Canteiras de Galicia (ACG), Galega de Graniteiros (AGG), de Maquinaria para la Piedra (GALIMAC) y de Marmolistas.

Las empresas que integran el Clúster del granito facturaron $336 \mathrm{M} €$ en 2011, siendo la industria gallega del granito la segunda más importante de Europa (tras Italia) y la quinta del mundo, por delante de potencias como Bélgica, Portugal o Alemania, y con cifras de exportación en 2011 de 85,8 M\$.

Según el Clúster del Granito, Galicia es la comunidad líder en España en producción de granito en bruto, la mayor parte del cual se transforma en las fábricas locales, que cuentan con la tecnología más avanzada. El resto se destina a la exportación.

Atendiendo a la Estadística Minera de España más reciente (MINETUR), la industria gallega del granito para todos sus usos (dicha estadística diferencia dos categorías: "granito ornamental" y "otros usos") queda retratada durante el año 2014 por las siguientes cifras: 94 explotaciones activas (de las 189 españolas) daban empleo directo a 798 personas, con una producción de más de 5 millones de toneladas (el $61 \%$ del total extraído en España) y un valor superior a los 47 millones de euros (el $56 \%$ del total español).

Centrando ahora la atención en las explotaciones de granito ornamental (excluyendo pues aquellas destinadas a "otros usos"), Galicia contaba con 53 explotaciones en 2014, responsables de 388 empleos directos, con una producción de 423.157 toneladas y un valor superior a los 23 millones de euros. Estas cifras representan, nuevamente, más del $50 \%$ del total español en cuanto a número de explotaciones, empleo, producción y valor del granito ornamental.

En cuanto a la provincia de Pontevedra, en ella se extrae cerca del $60 \%$ del granito gallego, tanto en tonelaje como en valor. En 2014, las 32 explotaciones de granito ornamental activas (la mayoría en la zona de O Porriño) daban empleo directo a 226 personas y produjeron 256.295 toneladas con un valor de $14 \mathrm{M} €$ (MINETUR).

Aunque los datos disponibles más recientes datan de 2014 (MINETUR) e indican un decrecimiento en los datos básicos de la extracción de granito ornamental en Galicia, se mantiene su importancia socio-económica tanto en la provincia de Pontevedra, como para Galicia y España.

Del yacimiento de "Rosa Porriño", en O Porriño, se extrae granito ornamental (para interiores y exteriores) en bloques, piedra de construcción y áridos de machaqueo, fundamentalmente para hormigones.

La roca ornamental se destina tanto al mercado nacional como internacional, comercializándose en un gran número de países (obras emblemáticas realizadas con este granito son, entre otras, el Ayuntamiento de Tokio y la sede del Parlamento Europeo en Bruselas). Portugal, Italia y Polonia son ahora los principales destinos de los bloques de granito gallego.

Por otra parte, y según los datos del Clúster del Granito, Galicia también es líder en la transformación del granito en España. Los 11 millones de $\mathrm{m}^{2}$ de productos elaborados en Galicia cada año representan el $85 \%$ del total de la producción española.

La industria transformadora del granito en Galicia es de gran importancia ya que en ella se elaboran (sobre todo bloques) unas 600.000 toneladas anuales, la mayor parte procedente de O Porriño, pero también cerca de 250.000 toneladas de materiales importados (granito s. I.) de países como Portugal, Angola, Sudáfrica, India o Mozambique.

En el año 2012 las exportaciones de granito de Galicia se incrementaron un $11 \%$, orientándose a nuevos mercados, y suponen el $73 \%$ de las exportaciones españolas. De ellas, el $95 \%$ se producen en la provincia de Pontevedra.

La importancia industrial del granito de Galicia se inicia en los años de 1960 y se debe en gran medida al yacimiento de granito "Rosa Porriño", objeto de la investigación (Baltuille et al, 2004) realizada por el Instituto Geológico y Minero de España (IGME) y la Escuela Técnica Superior de Ingeniería de Minas (ETSIM) (Taboada et al., 2004), de la Universidad de Vigo, como contribución al "Proyecto de Explotación" (Delgado y Ucha, 2004) a desarrollar por la empresa concesionaria Porriñesa de Canteiras, S.A. (POCASA), en la concesión de explotación "Benedicta" n 1.544, que ocupa su zona principal.

El carácter singular del yacimiento, la calidad contrastada del material obtenido, la atomización de la operación minera, realizada mediante numerosas unidades de explotación, y la proyección nacional e internacional de los productos obtenidos en los talleres de elaboración a partir, sobre todo, de la variedad de granito explotada con denominación "Rosa Porriño", fueron algunas de las razones que animaron 
a ambas instituciones a implicarse en la realización del trabajo que de un modo sintetizado se presenta en este artículo.

\section{Objetivos}

En el marco de los objetivos planteados en el Proyecto de Explotación requerido por la Administración según se establece en la Ley de Minas de 1973, para la renovación por otros 30 años de la concesión de explotación Benedicta $n^{\circ} 1.544$, se concretan los objetivos generales que corresponden a la parte del estudio que aquí se presenta y que se refiere a la obtención de la información geológica y minera necesaria para la evaluación de recursos de granito con posibilidad de explotación técnico-económica favorable.

Por tanto, el primer objetivo general planteado fue la realización del estudio geológico del yacimiento de granito Rosa Porriño y otras variedades inmediatas en su entorno, complementado con un segundo objetivo general consistente en la evaluación de los recursos de granito del yacimiento en explotación.

Como objetivos específicos se estableció la realización de:

- La cartografía geológico-minera de detalle (E. 1:3.500), por calidades industriales, como base para el cálculo de reservas del área actualmente en explotación y su entorno inmediato.

- La cartografía geológico-minera a E. 1:10.000 para la totalidad de la Concesión de Explotación "Benedicta".

- Un modelo digital 3D del área de explotación, al que se incorpore la cartografía de calidades industriales $y$ otra información geológica $y$ minera.

- La cubicación del yacimiento aplicando técnicas geoestadísticas.

- Los correspondientes informes de trabajos realizados y resultados obtenidos.

\section{Zona de estudio}

Los terrenos incluidos en la concesión minera "Benedicta" ( $\left.{ }^{\circ} 1.544\right)$ quedan en su práctica totalidad situados en el término municipal de O Porriño, y sólo el sector más oriental queda en el término de Salceda de Caselas, ambos en la parte suroeste de la provincia de Pontevedra. El área en explotación ocupa la parte más occidental de la concesión afectando (Fig. 1) a una superficie próxima a unos $2 \mathrm{~km}^{2}(2 \mathrm{~km}$ de largo según una orientación NNO-SSE y $1 \mathrm{~km}$ de ancho), del total de los $6.829 .462,83 \mathrm{~m}^{2}$ que comprende la concesión.

La concesión ocupa parte de un macizo montañoso que se eleva hasta poco más de los $400 \mathrm{~m}$ en Campanol y en O Faro, que constituyen junto con Faro de Budiño (399 m) una zona elevada y estrecha con fuerte relieve entre los cursos submeridianos del río Caselas por el Este y el Regato do Pozo da Regadeira por el Oeste. El resto de la concesión presenta un relieve acusado que desciende desde la cota de $327 \mathrm{~m}$ (O Corvo) hasta el entorno de los 30 a $40 \mathrm{~m}$ en la parte occidental de la concesión.

Debido a que la litología presente en la concesión es granítica, la morfología del terreno está condicionada por ella y por la red de fracturación, teniéndose un desarrollo importante de bolerío de diverso tamaño pero en general de orden métrico a decamétrico, o pequeñas cúpulas decamétricas.

La morfología natural del terreno está fuertemente modificada en el área de explotación, en la que las excavaciones afectan desde cotas del orden de los $250 \mathrm{~m}$ hasta los $17 \mathrm{~m}$ en los fondos de corta más occidentales. Los taludes de excavación son subverticales y llegan a tener en algún caso extremo hasta 70-80 m de desnivel.

Solamente el río Casavella en el ángulo NO de la concesión y el Arroyo do Pozo da Regadeira discurren por terrenos del derecho minero. La existencia de agua subterránea en la zona es escasa (Fonte de Santiago, Fonte Maceira), como puede constatarse por la escasa presencia y la poca importancia de las surgencias cortadas por las explotaciones.

El clima es de carácter atlántico con componente meridional, con veranos secos y calurosos. Las temperaturas son suaves (media anual de $14.8^{\circ} \mathrm{C}$ ) y las precipitaciones abundantes $(1.514 \mathrm{~mm}$ de media anual), repartiéndose en 105 días de lluvia al año. Son abundantes las nieblas.

O Porriño constituye una zona industrial de gran importancia y su gran desarrollo a partir de la década de los años sesenta trajo como consecuencia un fuerte incremento poblacional y la expansión de la actividad industrial de Vigo en el eje Vigo - O Porriño. Desde el eje local de comunicaciones que es la carretera de Salceda de Caselas, los accesos al área de explotación actual son numerosos y consisten en pistas que presentan algunos tramos asfaltados.

Desde el punto de vista geológico, el yacimiento se encuentra en el macizo granítico de O Porriño (Fig. 2 ), en la parte SO de la provincia de Pontevedra (Galicia) y se extiende hacia el sur, penetrando en Portugal. Su forma es subcircular, con cierto alargamiento N-S, unos $25 \mathrm{~km}$, y con anchuras E-O, que disminuyen desde su parte septentrional $(15 \mathrm{~km})$ hacia 


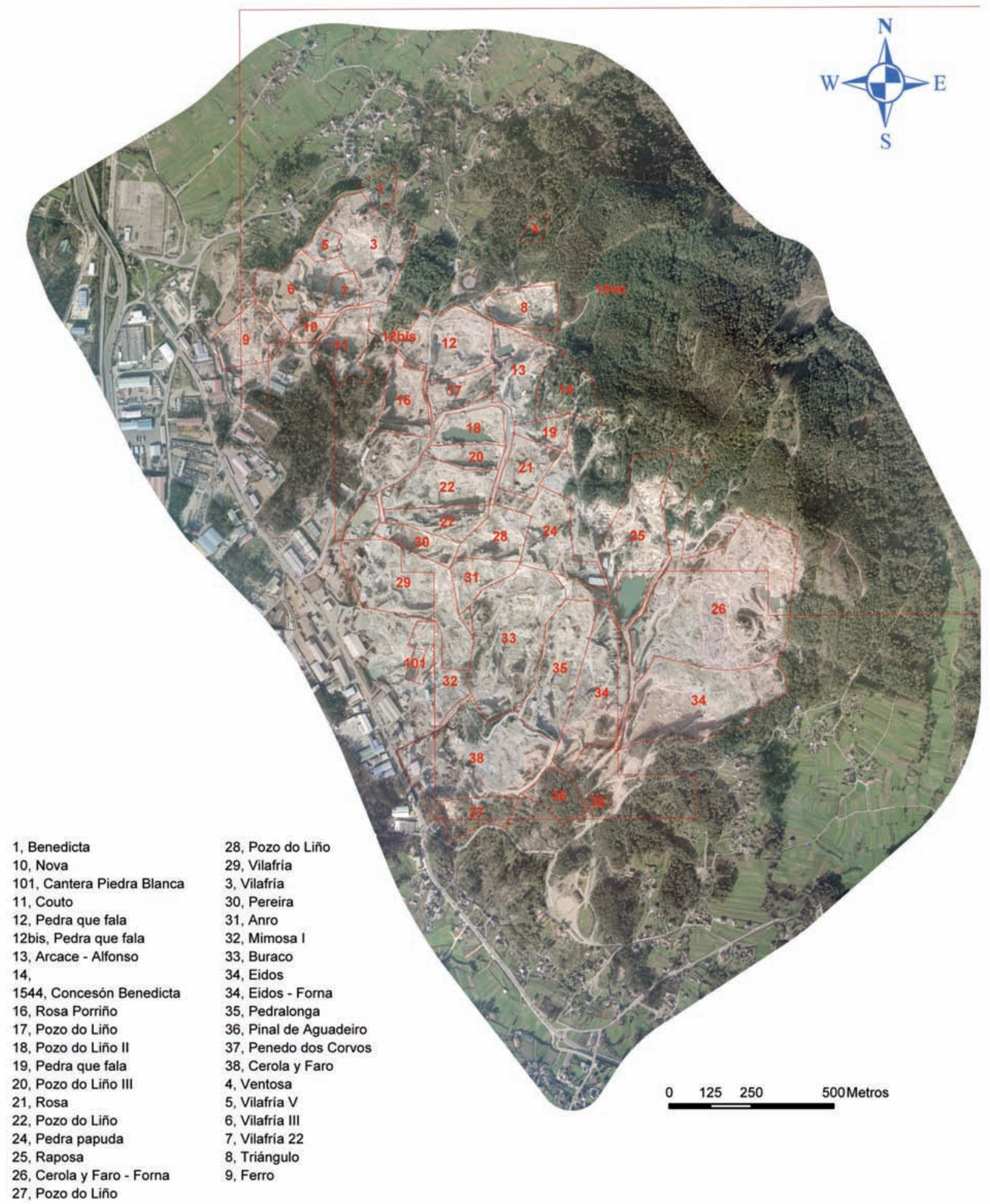

Figura 1. Canteras en la Concesión de Explotación "Benedicta".

Figure 1. Quarries in the "Benedicta" exploitation concession.

su parte meridional (10 km). El Macizo de O Porriño está emplazado en la Zona de Galicia - Trás-osMontes, representada en este sector por los materia- les esquistosos y gnéisicos de la Unidad Malpica-Tui. Con referencia a los tipos de granitoides de Galicia y del Macizo Ibérico, quedaría encuadrado en el grupo 


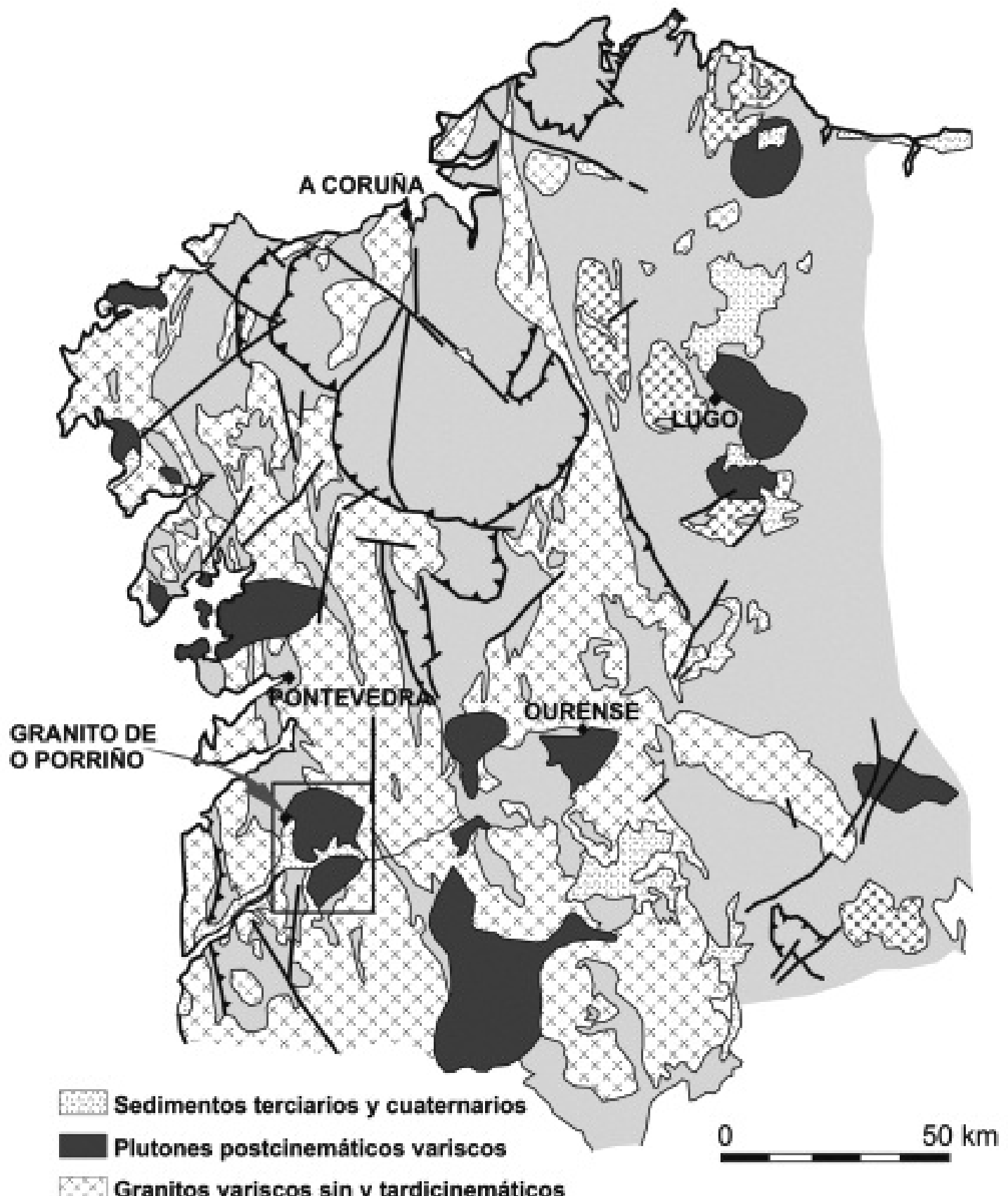

Granitos variscos sin y tardicinemáticos

Metasedimentos y unidades de los complejos alóctonos

Bellido et al. (2005)

Figura 2. Situación geológica del Macizo de O Porriño.

Figure 2. Geological situation of the O Porriño Massif.

de los granitoides postcinemáticos de emplazamiento somero (epizonal), que se caracterizan, desde el punto de vista estructural, por su emplazamiento posterior a todas las fases deformativas principales de la Orogenia Varisca y a los desgarres tardíos (Bellido et al., 2005).

\section{Metodología}

Para llevar a cabo el estudio geológico de detalle planteado en los objetivos 1 y 2 , se siguió la metodología de trabajo explicada a continuación, que tiene en cuenta la metodología general aplicada al estudio 
de macizos graníticos para su aprovechamiento como roca ornamental (Muñoz de la Nava et al., 1989), pero adaptándola a las especiales condiciones de una zona ya en parte intensamente explotada, con un conocimiento contrastado de las características tecnológicas del granito y con la posibilidad de una buena observación de las características litológicas y estructurales del yacimiento.

Por ello se ha tenido en cuenta la experiencia adquirida durante años de trabajo y comercialización del granito "Rosa Porriño" y se ha realizado un trabajo de observación sistemática y de detalle de los distintos frentes o canteras abiertas. Se ha realizado un esfuerzo integrador de los datos de calidad conocidos y observados en los levantamientos en campo con objeto de obtener una cartografía detallada de calidades industriales, para lo que se establecieron, después de los primeros trabajos, varias categorías de calidad que se fueron modificando y redefiniendo según avanzaban los trabajos, a fin de obtener una información cartográfica con calidades asignadas a los diferentes recintos cartografiados factibles de ser tratados en la fase de evaluación de recursos.

La metodología seguida ha incluido el análisis de documentación existente sobre el yacimiento, tanto de la facilitada por la concesionaria como la existente en el IGME, así como publicaciones u otros trabajos relacionados directa o indirectamente con el estudio. Se puso especial énfasis en la localización de sectores de características diferentes en el ámbito de la zona a estudiar, y en las características y parámetros a recoger durante el trabajo de campo, así como a su forma de representación.

Se abordó a continuación la realización de la cartografía geológico-minera del área de explotación a escala 1:3.500. Para ello se utilizó la fotografía aérea a escala $1: 5.000$ y la cartografía digital a escalas tanto 1:1.000 como 1:3.000, ambas en formato Cad-2D. Para el entorno inmediato a la zona de explotación se utilizaron también las fotografías aéreas 1:20.000 y bases topográfica 1:10.000.

Los trabajos se desarrollaron según los siguientes puntos:

\section{Delimitación de Dominios Homogéneos}

Se delimitaron sectores en los que los parámetros de interés para el cálculo de reservas fuesen lo más homogéneos posibles. Se consideraron como parámetros fundamentales la fracturación y la calidad industrial de la roca (Ferrero et al., 2005).

La fracturación condiciona la extracción de volúmenes y sus dimensiones, y como consecuencia su uso (ornamental, piedra de construcción o áridos). En los primeros recorridos de campo se observó que una mayor eficiencia cartográfica podría obtenerse si se integraban las características de fracturación en dos categorías: "Bandas de fracturas" y "fracturas".

La calidad industrial de la roca es bien conocida en el área, por lo que no se planteó la realización de ensayos tecnológicos de caracterización. La asignación de un valor de calidad de roca se ha basado en la observación de los frentes de cantera y en el conocimiento aportado por los canteros. La calidad industrial asignada a cada dominio homogéneo se obtuvo por integración del valor de la calidad de la roca y la fracturación, sistemática o no, observada en el dominio considerado.

Los trabajos de campo se realizaron sistemáticamente en todo el área de extracción, mediante la observación continua de los distintos taludes de explotación. Los datos obtenidos se representaron sobre la fotografía aérea 1:5.000, en la que además de la fracturación cartografiable se incluyó un código de calidad industrial para cada dominio considerado homogéneo.

Para disponer de datos útiles para conocer la estructura en vertical del yacimiento en la parte visible, las observaciones de los distintos sectores se integraron en estaciones de observación, realizándose un total de 30 . En ellas se sintetizan las variaciones verticales y los aspectos de interés relativos a la fracturación así como a la calidad de la roca con referencias a la mineralogía, alteraciones, color, tamaño de grano, textura, estructura, fisuración, venas, enclaves, bolsadas, estructuras de flujo, y a la distribución de estas características. Se incluye en ellas una codificación para la fracturación y otra para la calidad; así como el código estimado para la calidad industrial del macizo rocoso en cada tramo delimitado.

Todos los datos sobre parámetros de interés adquiridos en las distintas estaciones de observación en canteras se incluyeron en una base de datos para facilitar su análisis. La cartografía de calidades y cortes de referencia a través del yacimiento se realizaron utilizando metodología SIG, con Arcview y compatibilidad con Geomedia.

\section{Sondeos mecánicos}

Con objeto de completar la información en vertical del yacimiento en la parte actualmente no accesible se realizaron 5 sondeos mecánicos $(169,25 \mathrm{~m}$ perforados) con recuperación de testigo, situados en los fondos de corta de canteras de "Rosa Porriño". Otros dos sondeos de $81 \mathrm{~m}$ y $54,10 \mathrm{~m}$ se ubicaron en la 
Ferrero Arias, A., et al., 2017. Aplicación práctica de la metodología de caracterización... Boletín Geológico y Minero, 128 (2): $451-466$

facies "Rosa Porriño porfídico". Los sondeos se testificaron y el resultado se representó en las correspondientes columnas a escala 1:100 y en los perfiles geológicos del yacimiento.

\section{Estudio de fracturación}

Como consecuencia de la discretización de la deformación del macizo granítico, la fracturación sistemática fuera de las bandas de fracturación señaladas en la cartografía es escasa y con espaciados muy amplios. Ello, unido a que el corte se realiza en la práctica totalidad de los casos con hilo, hizo que no se planteara la realización de estudios de blocometría. Sin embargo, sí interesaba conocer el patrón de fracturación a escala del área de estudio, así como su encuadre en la estructura general de la zona. Además, el análisis de la fracturación permite profundizar en el conocimiento de los diferentes sistemas de fracturas existentes y sus relaciones, base para intentar enfatizar las direcciones de fracturación más persistentes y que afectan más a la explotación de los recursos de granito ornamental. También permite conocer la cinemática de las principales fallas que cortan el área de explotación.

Para obtener los resultados esperados del análisis de fracturación se han analizado espacialmente las trazas de fracturas (discriminando entre bandas de fracturas y fracturas), realizando 8 transversales en las que se han medido además de la dirección y buzamiento, el espaciado entre fracturas y su apertura, así como su tipo de relleno; se han medido también estrías en los planos de fallas. En el análisis de transversales se ha hecho una discriminación entre fallas y diaclasas. Con objeto de conocer mejor la disposición del "andar" en la zona de extracción actual, se realizaron medidas de cortes con hilo y se analizó su distribución (en la industria extractiva del granito ornamental se denomina "andar" al plano del macizo o del bloque extraído que se considera más favorable para el aserrado en el taller).

Todos los datos obtenidos en el terreno han sido informatizados y tratados, utilizando varios programas específicos de geología estructural y estadística como por ejemplo, Cratos vs.1.0, TectonicsFP vs. 1.5, StereoNet vs. 3.0, Geocalculator vs. 4.0, Joints vs. 1.10. y Statistica vs. 6.0.

\section{Cartografía geológico-minera de "Benedicta"}

La cartografía de calidades industriales de la zona de explotación se complementó con la realización, utilizando metodología SIG con Arcview, de la cartografía geológico-minera de la concesión "Benedicta". Se realizó esta cartografía siguiendo los métodos habituales, basados en las observaciones de campo con apoyo de la foto aérea y la base topográfica digital a escala 1:10.000.

Se hicieron trabajos de fotointerpretación, de reconocimiento de campo y un estudio petrológico para establecer las diferentes facies graníticas y sus relaciones en campo. Se tomaron datos en 38 estaciones de observación repartidas por toda la concesión "Benedicta", pero con mayor densidad en el área de explotación, observando la naturaleza litológica de la roca y su estado de alteración y fracturación. Asimismo se tomaron 7 muestras en distintas facies de granito para su estudio en lámina delgada y 4 muestras para análisis químico general, con objeto de una mejor caracterización de la roca.

\section{Evaluación de recursos}

Para obtener un modelo digital 3D del área de explotación, al que se incorpore la cartografía de calidades industriales y otra información de interés aportada por el estudio geológico y minero del yacimiento se empleó un método innovador especialmente diseñado para batolitos de gran tamaño, tomando como unidad base de cálculo bloques cúbicos de $1.000 \mathrm{~m}^{3}$ (del mismo tamaño que los bloques primarios extraídos en cantera).

Una vez conocida la calidad industrial de la roca (calidad tecnológica y estética), la fracturación es el principal parámetro a la hora de definir la calidad comercial del granito, por lo que se definieron cuatro calidades en función de su grado de fracturación: la mejor calidad corresponde a volúmenes de calidad ornamental óptima que permiten la obtención de bloques de 6-12 $\mathrm{m}^{3}$, aptos para su corte en telar; la segunda calidad denota volúmenes de calidad ornamental adecuada, aunque el máximo tamaño de bloque extraíble es de $6 \mathrm{~m}^{3}$, válido para el corte con telar, con un espaciado de discontinuidades mayor de 2 metros; una tercera calidad se asigna a aquellos volúmenes de roca aptos para mampostería, de los cuales se podrán obtener semibloques de menos de $4 \mathrm{~m}^{3}$, con un espaciamiento entre discontinuidades menor de 2 metros; por último, la cuarta calidad corresponde a aquellos volúmenes sólo aptos para su aprovechamiento como áridos de construcción, con una fracturación más intensa.

Para la determinación de las reservas de las distintas calidades se aplicó el kriging difuso o fuzzy kriging (Zadeh, 1965), una técnica geoestadística que está 
especialmente indicada para problemas con un cierto grado de incertidumbre asociado. Un conjunto difuso, $A$, definido para el conjunto de números reales $x$, es un conjunto de pares ordenados:

$$
A=\{[\mathrm{x}, \mu(\mathrm{x})]\}
$$

donde $\mu(x)$ es la función de pertenencia para $x$ en $A$ en el intervalo $[0,1]$, indicando los valores cercanos a 0 un bajo grado de pertenencia, y los cercanos a 1 un alto grado de pertenencia. Un número difuso es un conjunto difuso del conjunto de números reales con una función de pertenencia convexa que satisface que $\mu(x)=1$ en al menos un punto.

El método aplicado en este trabajo (Taboada et al., 2004) incluye el uso del kriging convencional con información difusa, adaptando la teoría de conjuntos difusos al modelo para asignar clases de calidad a un bloque primario de $1.000 \mathrm{~m}^{3}$ de acuerdo con el nivel de fracturación. Dado que el grado de fracturación de un bloque de tales dimensiones es heterogéneo, tanto en intensidad como en espaciado, dirección, etc., la asignación de un solo grado de fracturación conllevaría a unas estimaciones económicas incorrectas. Además, la frontera entre las distintas clases es difusa, siendo el cambio de una a otra gradual, lo que implica un grado de incertidumbre en la definición de calidades y por tanto un error de estimación, que en todo caso será siempre menor que el error cometido al asumir una única calidad para el bloque primario.

Empleando un procedimiento basado en la teoría de conjuntos difusos, se aplicó una función triangular para la asignación de calidades de los datos de entrada y de los resultados del modelado. Las funciones de pertenencia, muy empleadas en la teoría de conjuntos difusos, y los números difusos se emplearon por su simplicidad computacional y su flexibilidad para reflejar la información disponible.

Para cada bloque de granito de $1.000 \mathrm{~m}^{3}$ se cons-

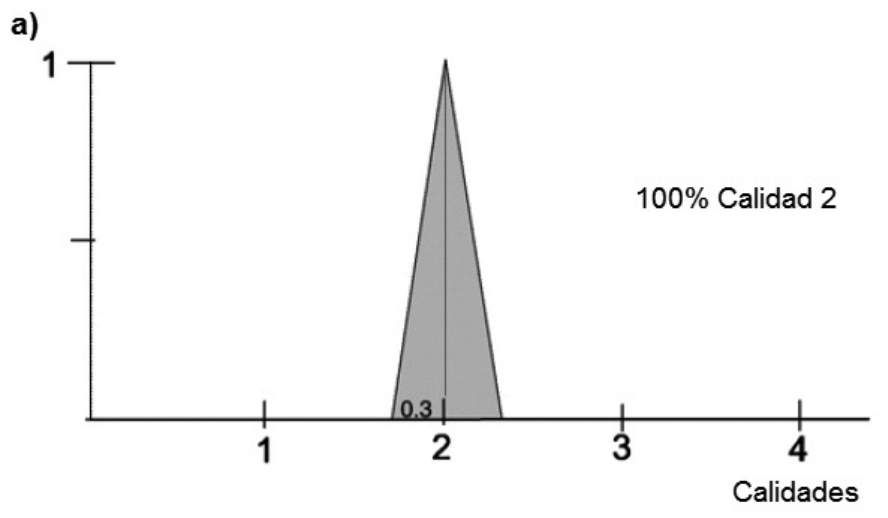

b)

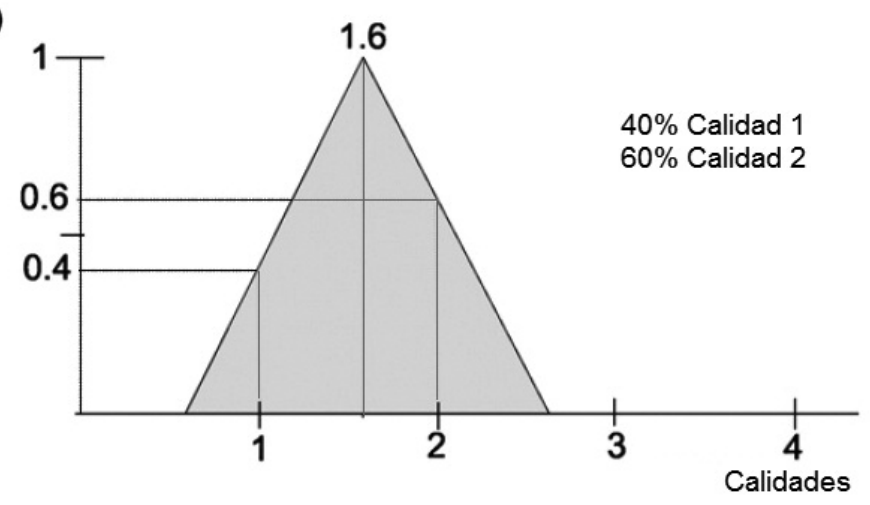

c)

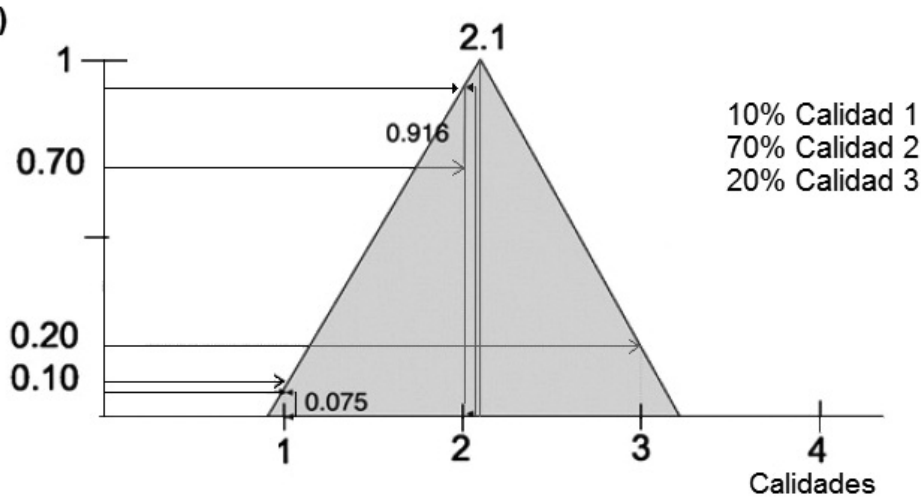

Figura 3. Ejemplos de funciones de pertenencia triangulares para los porcentajes de calidad definidos para tres bloques de (a) una calidad comercial, (b) dos calidades comerciales y (c) tres calidades comerciales.

Figure 3. Examples of triangular membership functions for quality percentages defined for three different blocks of (a) a single commercial quality, (b) two commercial qualities and (c) three commercial qualities. 
truyó un triángulo que refleja su distribución de calidades de la siguiente manera: el eje $\mathrm{X}$ representa las distintas clases comerciales, y el ejeY el porcentaje de cada clase presente en el bloque (Fig.3). En el caso de un bloque de tres calidades, para la adaptación a la función triangular es necesario recalcular los porcentajes de cada calidad, como se muestra en la Fig. 3.c.

El kriging forma parte del grupo de estimadores espaciales tradicionalmente empleado con datos concretos (Journel y Huijbregts, 1978), mientras que el kriging difuso es una generalización de los métodos de kriging que emplean datos tanto concretos como con un cierto nivel de incertidumbre. Empleando la metodología desarrollada por Diamond (1989) y adaptándola a datos anisótropos, se estimó la distribución espacial de los números difusos y se calcularon las varianzas de la estimación. Para ello, se modeIaron los datos triangulares difusos con los variogramas tipo lower crisp, modal crisp y upper crisp, resultantes de los valores de los vértices izquierdo, superior y derecho de los triángulos, respectivamente. Mediante la resolución de un sistema de tres funciones, para cada localización de interés $x$ se calcula la distribución de calidades, y con el vector de pesos $\left(\lambda_{1}(x), \lambda_{2}(x), \ldots, \lambda_{n}(x)\right)$ se calcula un estimador $(x)$, que es el valor difuso estimado para la posición $x$ :

$$
Z(x)=\sum_{i=1}^{n} \lambda_{i}(x) \cdot Z\left(x_{i}\right)
$$

Los datos de entrada se referenciaron con coordenadas en las tres dimensiones de los puntos centrales de los bloques de $1.000 \mathrm{~m}^{3}$, asociando a cada uno un número difuso triangular que indica la calidad aproximada de todo el bloque. Los datos calculados se estimaron con el algoritmo de kriging, obteniendo los números difusos triangulares asociados a los puntos centrales de los bloques no observables.

Asimismo, para la representación del comportamiento anisótropo de las fracturas del macizo se aplicaron distintos semivariogramas direccionales con el fin de detectar los rangos de los semivariogramas experimentales, reflejando la dependencia espacial asociada a la dirección de fracturación principal.

\section{Resultados y discusión}

Los materiales que ocupan el área de estudio pueden agruparse en materiales del recubrimiento y las rocas graníticas del yacimiento. El recubrimiento lo forman depósitos detríticos (arenas y gravas) que se encuentran en los fondos de valle (aluviales) y los jabres o arenas graníticas (cuarzo feldespáticas) que forman depósitos eluvio-coluviales. Los espesores del recubrimiento suelen ser inferiores a los $5 \mathrm{~m}$, si bien pueden localmente (vaguadas en zonas fracturadas) superar los 10-15 m.

Las rocas graníticas que constituyen el Macizo de O Porriño presentan una morfología más general en bolos o en pequeñas cúpulas con fracturación subhorizontal bastante densa en la parte más superficial (hasta 20 ó $30 \mathrm{~m}$ en ocasiones) lo que favorece la profundización de la alteración meteórica, y dificulta o imposibilita la extracción de bloques comerciales.

El estudio petrológico y geoquímico realizado en la concesión "Benedicta", ha permitido determinar que los granitos de la facies "Rosa Porriño" se han emplazado posteriormente a los de la facies "Gris Mondariz", que pueden encontrarse como enclaves dentro de los primeros. También se ha puesto de manifiesto la presencia de una variedad porfídica de los granitos de tipo "Rosa Porriño" en la que el feldespato potásico tiene una tendencia general a formar megacristales.

Los estudios realizados permiten señalar que las características petrológicas (Bellido et al., 2005) y estructurales (Gumiel et al. 2006) de los granitos del Macizo de O Porriño, indican que su intrusión ha sido posterior al principal pico metamórfico regional y a los procesos de migmatización, así como la calma tectónica existente durante su emplazamiento y su carácter postcinemático.

Las facies graníticas que constituyen el Macizo corresponden a facies más básicas al este y facies más ácidas al oeste, indicando una evolución hacia rocas más evolucionadas hacia el Oeste. Así, en la parte oriental se tiene una facies de monzogranito porfídico más o menos anfibólico, que se ha denominado "Granito Gris Mondariz" (variedad comercial Gris Mondariz, que en algunos sectores presenta ciertas variaciones que constituyen la variedad comercial Rosa Dante). En la parte central de la zona estudiada aflora una facies de granito rosa porfídico que se ha denominado "Granito Rosa Porriño porfídico" y en la parte occidental, área de explotación actual, aflora una facies inequigranular homogénea de granito rosa (Fig. 4) que se denomina "Granito Rosa Porriño" (variedad comercial Rosa Porriño).

En relación con la fracturación, además de la marcada fracturación subhorizontal que la roca presenta en su parte más superficial, existe una fracturación con espaciado amplio a muy amplio (>10 m) (Fig. 5) y "bandas de fracturas" (Fig. 6), constituidas por fallas y diaclasas, en las que el espaciado interfracturas es bajo a muy bajo (inferior a $2 \mathrm{~m}$ y en general decimétricos). 


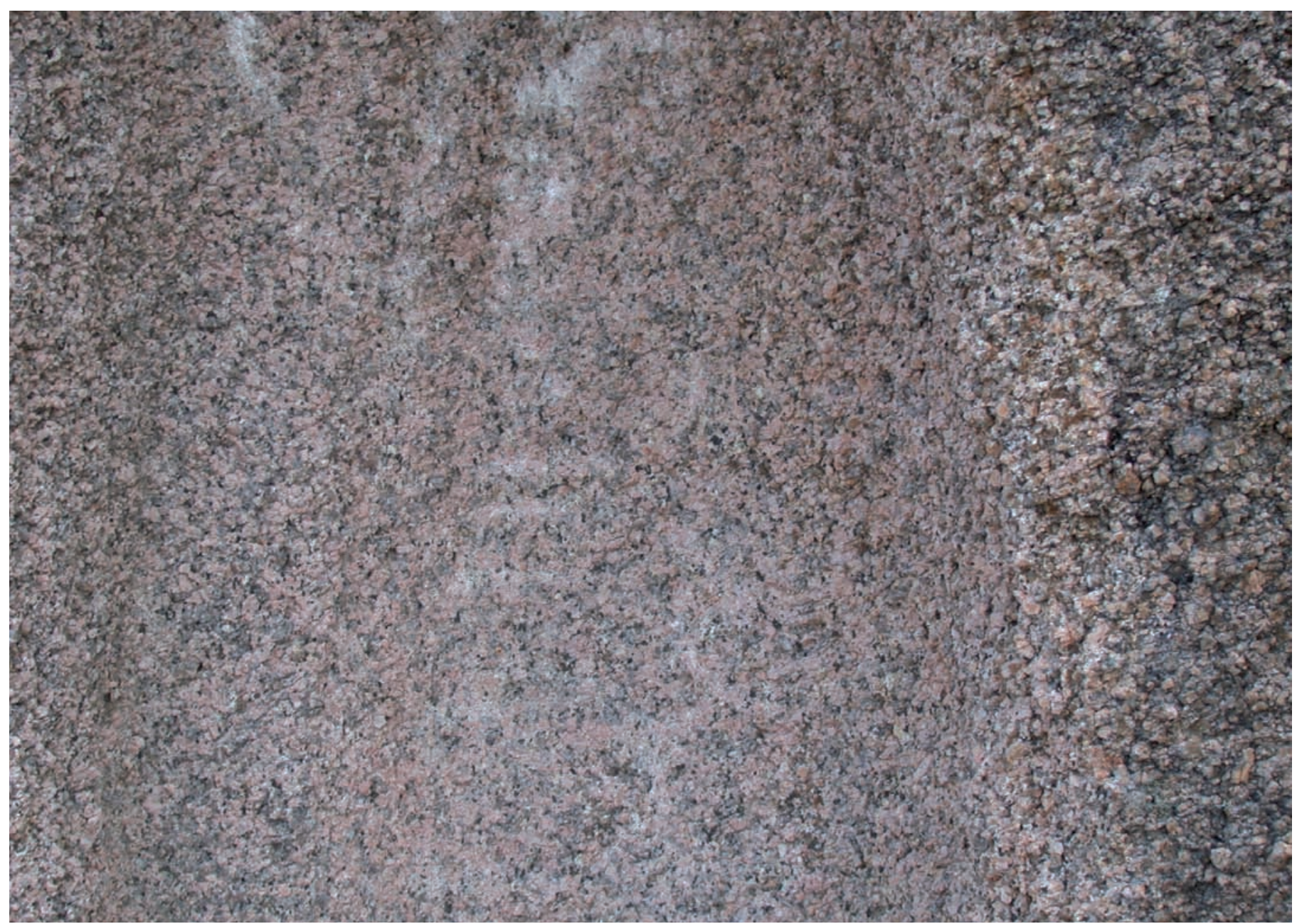

Figura 4. Aspecto textural de la variedad "Rosa Porriño".

Figure 4. Textural aspect of the variety "Rosa Porriño."

La orientación predominante de las fallas corresponde a dos grupos de fallas conjugadas de dirección NNE-SSO y NNO-SSE, aunque hay otros grupos de fallas de orientación NE-SO y NO-SE también conjugadas y finalmente otro de menor importancia de orientación E-O. Los buzamientos de los planos de fallas varían entre $70^{\circ}$ y $90^{\circ}$.

El patrón de fracturación es típicamente direccional-normal, con un máximo de compresión subhorizontal y de orientación aproximada N-S; y un máximo de extensión subhorizontal y de orientación aproximada E-O. Los ejes de esfuerzo máximo y mínimo son casi horizontales y el intermedio es prácticamente vertical, y su orientación es coherente con la orientación media de máximo acortamiento horizontal N176 ${ }^{\circ}$ obtenida.

La existencia de sectores donde los espaciados son amplios a muy amplios entre "bandas de fractu- ras" con espaciados bajos a muy bajos, obliga a una explotación, aunque condicionada por la fracturación natural, mediante corte artificial (corte con hilo). Estos cortes siguen direcciones sensiblemente submeridianas que se adaptan a la dirección del "andar" de la roca que se presenta con buzamientos entre $0^{\circ}$ y $10^{\circ}$ al Oeste, siendo lo más general $5^{\circ}$ Oeste. Si admitimos lo apuntado por algunos autores y lo observado en este trabajo de que el "andar" responde a una fisuración del granito más probablemente debida a enfriamiento y descompresión del macizo, podría indicar una cúpula inmediata al este de la zona donde se han realizado las medidas (parte centro oriental del área en explotación). Otro plano de corte se dispone perpendicular (sensiblemente E-O) y subvertical, estando el "levante" o bien el "andar" prácticamente horizontal.

Después de los primeros reconocimientos de 


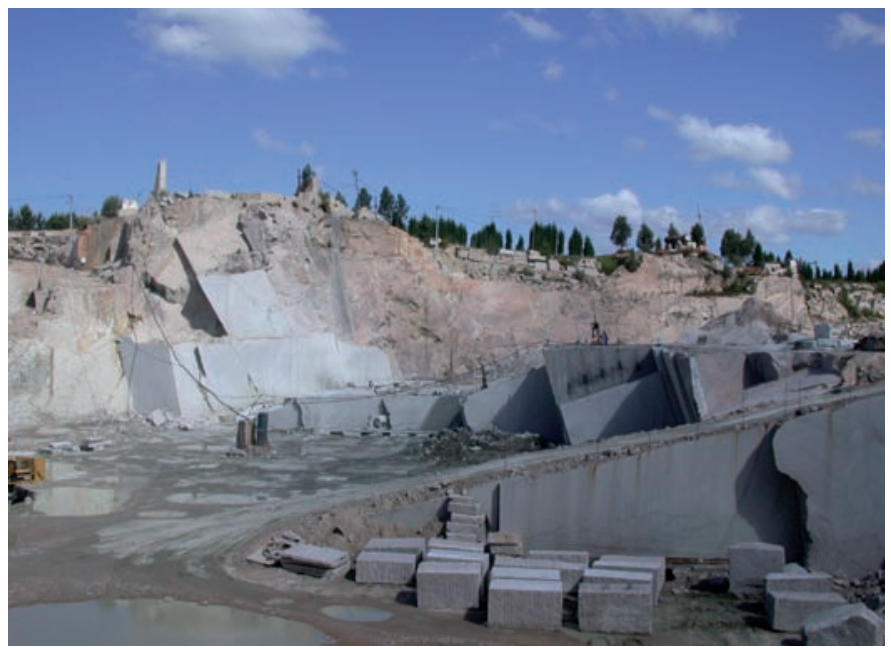

Figura 5. Fracturación con espaciado amplio y corte con hilo. Nótese el corte con talud invertido, condicionado por el "andar".

Figure 5. Wide-spaced, wire-cut fracturing. Note the inverted cut slope, conditioned by the "andar".

campo y consultas de datos de productores se llegó a la conclusión de que algunos de los parámetros comercialmente valorables (ligeras variaciones de color o brillo y otros) no podían trasladarse, en todos los casos, a la cartografía de calidades, mientras que otros podían ser considerados en algunos dominios (alta porosidad, heterogeneidades...). Finalmente se optó por asignar en la cartografía las cuatro calidades industriales ya definidas (calidad primera, segunda, tercera y cuarta) para cada dominio homogéneo.

A los distintos dominios homogéneos representados en la cartografía de calidades industriales (Fig. 7) se les ha asignado un color y código, lo que permite su tratamiento geoestadístico, previa adaptación de los términos mixtos.

La cartografía geológica de la zona de explotación y gran parte de la concesión "Benedicta" puede verse de forma simplificada en la Fig. 8, donde además de la distribución de las distintas facies graníticas mayoritarias, se ha incluido la red de fracturación y las estaciones de observación y la ubicación de sondeos.

A partir de los datos de campo organizados espacialmente e identificando los puntos centrales de los bloques de $1.000 \mathrm{~m}^{3}$ definidos en el yacimiento, se obtuvo la distribución de calidades mediante la técnica de kriging difuso tanto para toda el área de estudio como para distintos planos horizontales del depósito, desde la menor altitud definida por la base de los sondeos hasta la mayor altitud $(285 \mathrm{~m})$ del afloramiento.

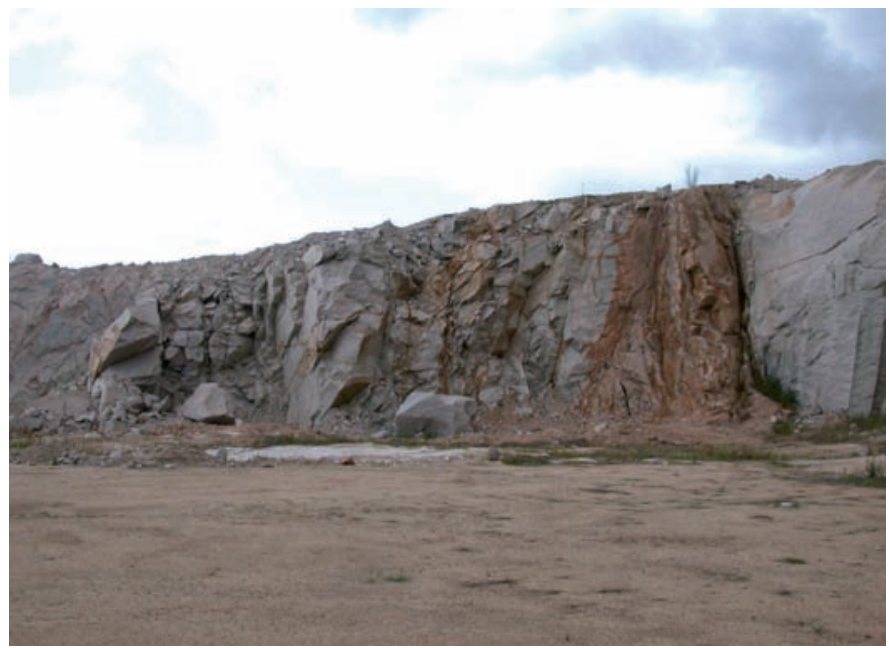

Figura 6. Aspecto de una de las bandas de fractura incluida en el estudio de fracturación.

Figure 6. Appearance of one of the fracturing bands included in the fracturing study.

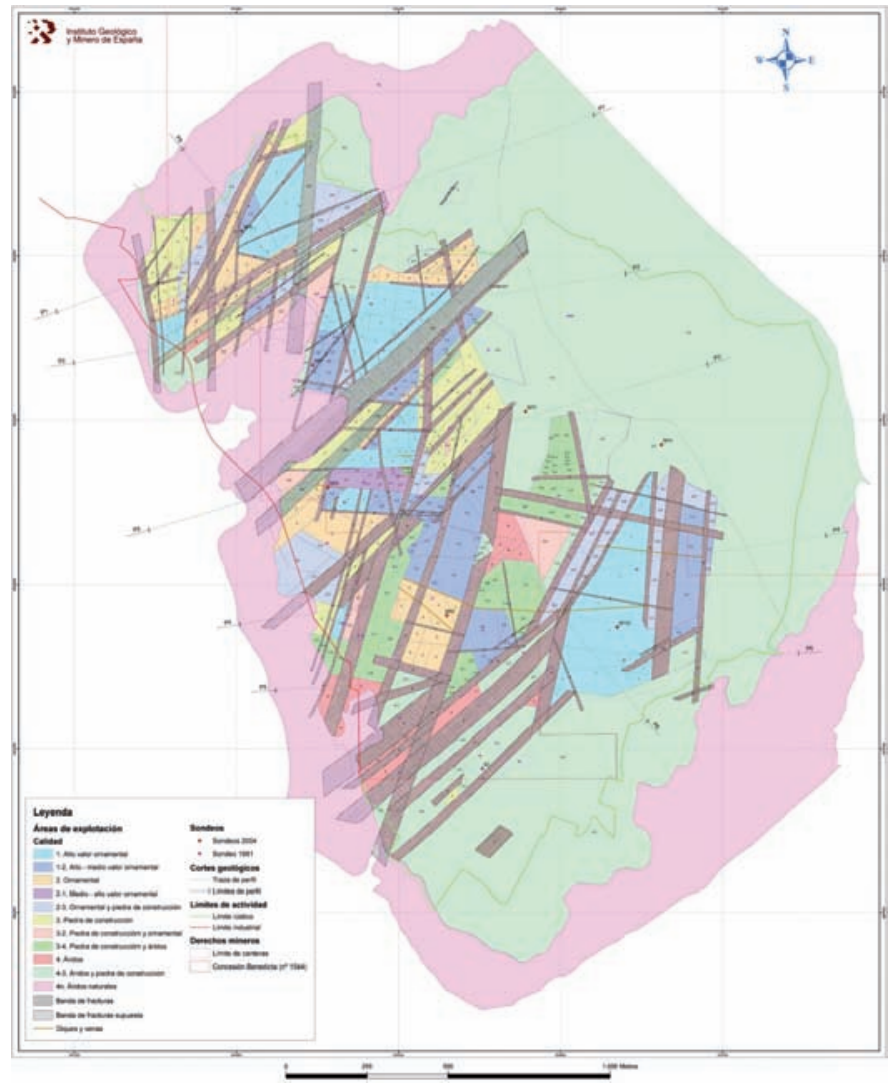

Figura 7. Cartografía de calidades industriales de la zona de explotación de granito "Rosa Porriño".

Figure 7. Mapping of industrial qualities of "Rosa Porriño" granite mining área. 


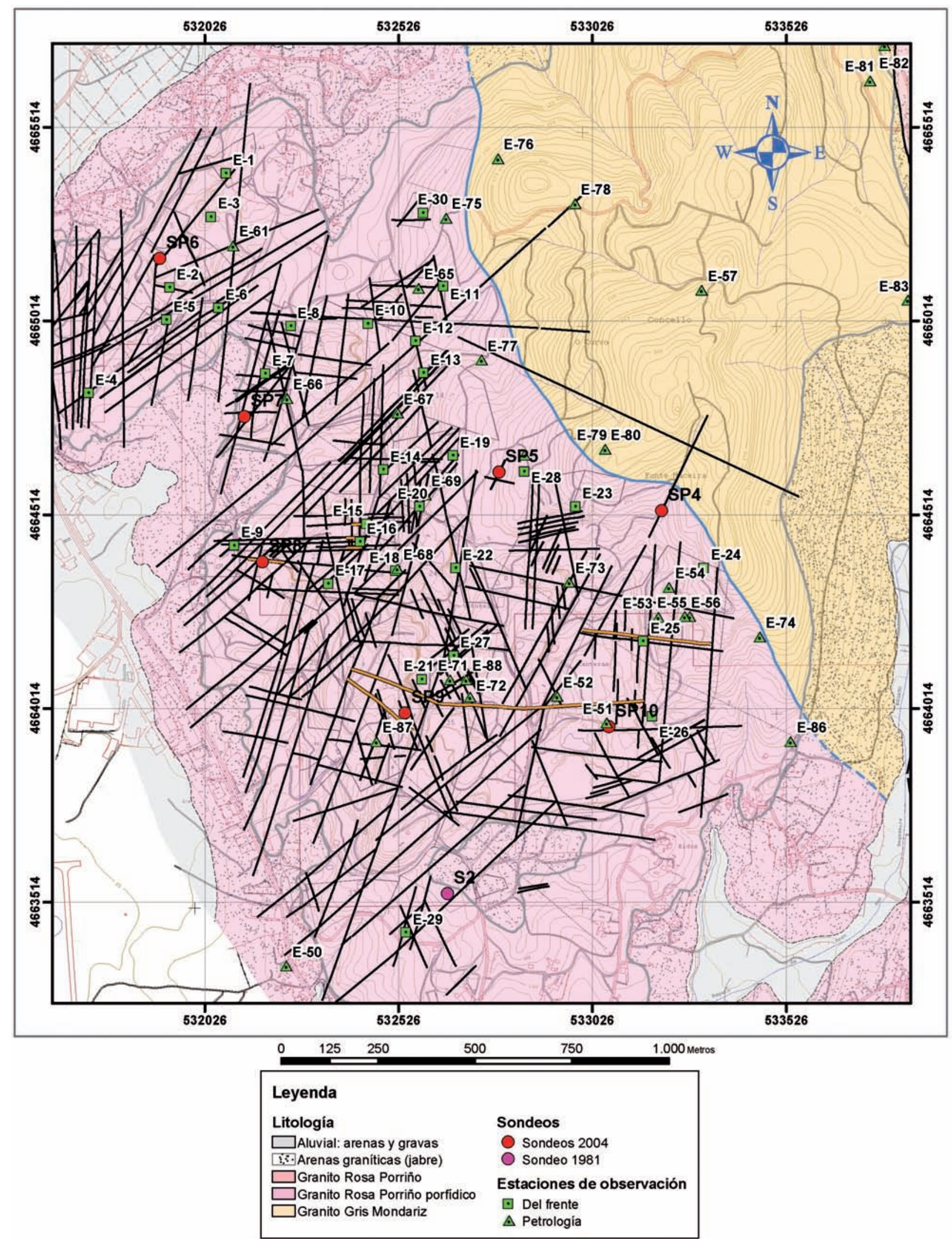

Figura 8. Estaciones de observación en el área de explotación, fracturación y cartografía geológica. Figure 8. Observation stations in the exploitation area, fracturing and geological mapping. 
De un volumen total de $124.109 .970 \mathrm{~m}^{3}$, se obtuvo la siguiente estimación de reservas: el 13,89\% corresponde a granito de primera calidad, el $15,96 \%$ a granito de segunda calidad, el $21,59 \%$ a la tercera calidad y el restante $48,56 \%$ a granito de cuarta calidad. Se vio que la distribución de calidades depende de la profundidad en el batolito, ya que las zonas más elevadas contenían menores cantidades de segunda calidad, más frecuente en zonas a mayor profundidad.

La Fig. 9 muestra la cubicación de reservas según calidades, con los bloques definidos y su calidad mayoritaria representada (no obstante, a cada bloque se le asignaron los porcentajes presentes de las cuatro calidades).

Los resultados del kriging se mejoraron con una aplicación gráfica consistente en la programación en Microsoft Database de una serie de consultas en lenguaje SOL para representar el conjunto de bloques del depósito en tres dimensiones, permitiendo a su vez la consulta de información detallada de cada bloque.

\section{Conclusiones}

El carácter postcinemático de los granitos del Macizo de O Porriño, determina en general una alta homogeneidad, escasa presencia de enclaves y ausencia de estructuras deformativas, por lo cual estos granitos presentan una idoneidad significativa para su utilización como roca ornamental de alto valor estético y técnico.

La variedad comercial Ilamada en trabajos anteriores Rosa Dante se ha venido asignando tanto a la facies definida aquí como "Rosa Porriño porfídico" como a la facies "Granito Gris Mondariz", si bien en general se ha relacionado con esta última facies. En el estudio realizado se indica la proximidad petrológica del Rosa Dante al Gris Mondariz y se individualiza la facies "Rosa Porriño porfídico", como facies petrológica más próxima a la que constituye la variedad comercial "Rosa Porriño".

La calidad industrial de los materiales que consti-

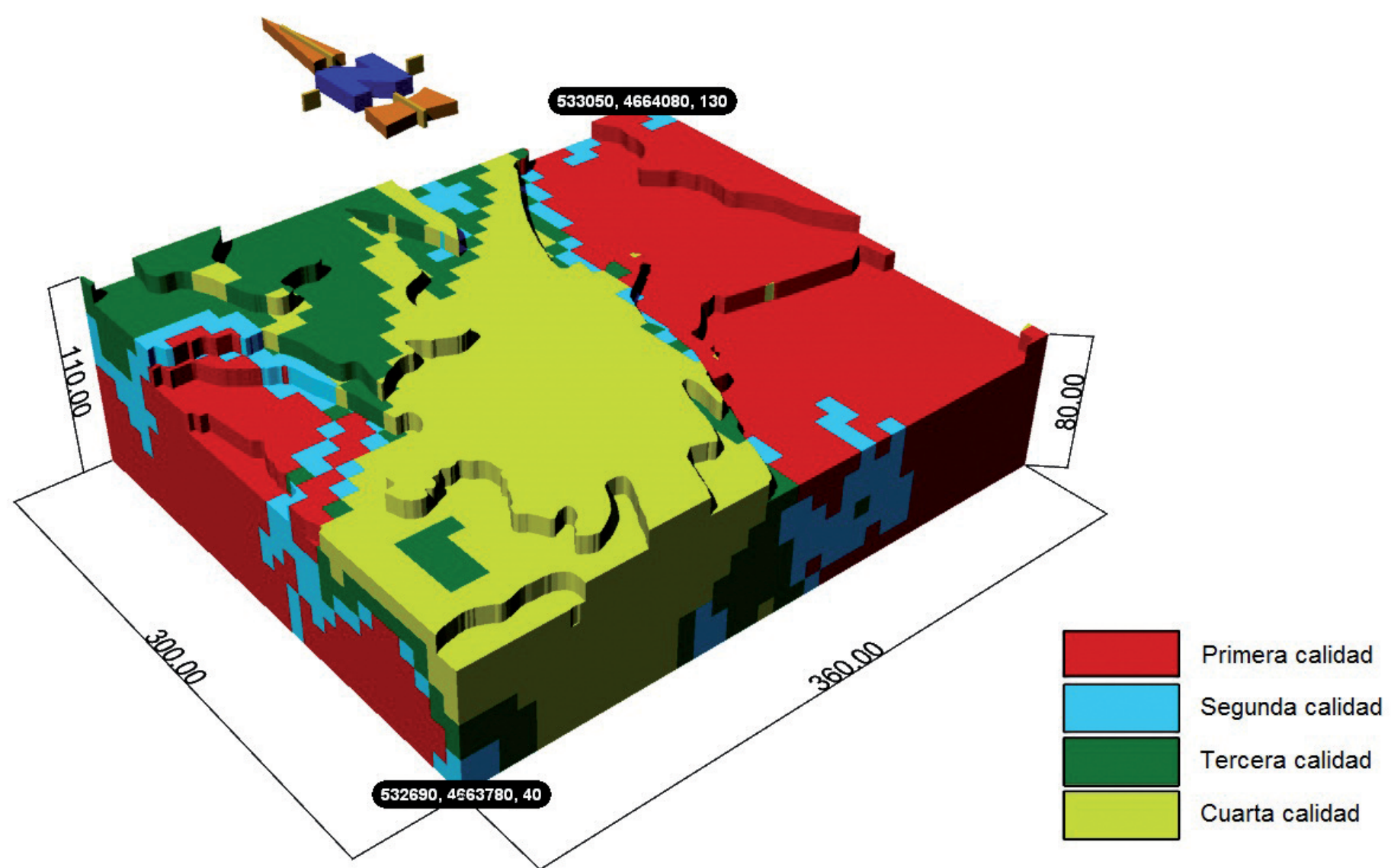

Figura 9. Vista del modelo del depósito resultante. Figure 9. Overview of the resulting deposit model. 
tuyen la concesión "Benedicta" puede agruparse, a la escala del trabajo realizado, con referencia a los productos que predominantemente pueden obtenerse. Se obtiene así una cartografía de calidades industriales que se ha mostrado muy útil para el cálculo de recursos, y con alta fiabilidad dado el carácter continuo de las observaciones que integra.

Se plantea la posible utilidad del análisis del espaciado empleado en este trabajo en la predicción del tamaño de bloque que podría obtenerse al avanzar en una determinada dirección y al nivel estructural del macizo en el que se realizan las medidas.

La orientación de los tensores de esfuerzos y la dirección aproximadamente N-S obtenida en el estudio de fracturación es coherente con el acortamiento $\mathrm{N}-\mathrm{S}$ de la corteza que se produjo durante la etapa compresiva alpina en la parte septentrional de la península, de edad Eoceno-Mioceno, a la cual asociamos la deformación frágil del Macizo de O Porriño.

La metodología aplicada para la estimación de reservas del yacimiento combinando la lógica difusa y las técnicas de kriging ha permitido analizar la distribución de las distintas calidades comerciales de granito ornamental de los bloques primarios definidos. Se considera que cada bloque primario de 1.000 $\mathrm{m}^{3}$ puede contener más de una calidad comercial, y se estiman los porcentajes de cada una de las cuatro calidades establecidas.

El enfoque basado en el kriging para la estimación de las calidades comerciales de roca ornamental posee dos características novedosas. Por una parte, el enfoque matemático difuso para la asignación de calidades refleja el hecho de que la fracturación afecta de una manera progresiva a los bloques, por lo que las fronteras entre las calidades no son claras. Además de esto, la fracturación a nivel de yacimiento es anisótropa, y dado que es el factor más determinante de la calidad de los bloques, este método permite la obtención de un estimador espacial real de la calidad.

La aplicación de esta metodología innovadora a uno de los depósitos más relevantes del mundo representa un avance en las técnicas existentes para la estimación de las reservas de roca ornamental, ya que proporciona unas estimaciones más realistas basadas en el método de explotación, la anisotropía del depósito y las fronteras difusas existentes entre las diferentes clases comerciales definidas.

\section{Referencias}

Baltuille, J.M., Ferrero, Á., Monteserín, V., Gumiel, P. y Bellido, F. y Araujo, M. 2004. Estudio geológico-minero de la concesión de explotación "Benedicta" ( $\left.n^{\circ} 1.544\right), 0$ Porriño (Pontevedra). En: Proyecto de prórroga de la C.E. "Benedicta" $n^{\circ}$ 1.544. Informe de POCASA-Porriñesa de Canteiras, S.A. (inédito). Tomo I, 225 pp., y planos.

Bellido, F., Monteserín, V., Gumiel, P., Ferrero, Á., Baltuille, J.M. y López, M.T. 2005. Características petrológicas y geoquímicas de las principales variedades de granitos ornamentales del macizo de "O Porriño" (SO de Galicia). Boletín Geológico y Minero, 116 (4), 331-349.

Clúster del Granito, 2013. Información básica corporativa 2013. Clúster del granito. Departamento de comunicación.

Delgado, J.L. y Ucha, M. 2004. Proyecto de prórroga de la C.E. "Benedicta" $n^{\circ}$ 1.544. Informe de POCASA-Porriñesa de Canteiras, S.A., 8 tomos (inédito).

Diamond, P., 1989. Fuzzy kriging. Fuzzy sets and systems 33, 315-332.

Ferrero, Á.; Monteserín, V.; Baltuille, J.M.; Gumiel, P. y Bellido, F. 2005. La información geológico-minera aplicada a la estimación de reservas de granito ornamental: El caso del yacimiento de "Rosa Porriño". Actas XVI Congreso Geológico Argentino, 2, 817-824. La Plata, Argentina. ISBN: 987-595-003-3.

Gumiel, P., Monteserín, V., Ferrero, Á., Bellido, F., Baltuille, J.M. y Vivar, V. 2006. Aplicación del análisis geométrico de fracturas a la determinación de bloques en las canteras de granito de O Porriño, Pontevedra (España). Revista de la Sociedad Geológica de España, 19 (1-2), 25 $\mathrm{pp}$.

Journel, A.G., Huijbregts, C.J., 1978. Mining geostatistics. Academic Press, New York.

MINETUR. Estadística Minera de España 2014. Publicaciones del Ministerio de Industria, Energía y Turismo (MINETUR), Madrid. Disponible en www.minetur.gob.es

Muñoz de la Nava, P., Romero Escudero, J.A., Rodríguez Suárez, I., García Romero, E., Crespo Rosa, A., Carrión Moles, F. y Garbayo Martínez, M.P. 1989. Metodología de investigación de Rocas Ornamentales: Granitos. Boletín Geológico y Minero, 100 (3), 433-453.

Taboada, J., Saavedra, A., Giráldez, E., Fernández de la Vega, D., y Domínguez, R.A., 2004. Evaluación de recursos de la concesión de explotación Benedicta $n^{\circ} 1.544,0$ Porriño (Pontevedra). En: Proyecto de prórroga de la C.E. "Benedicta" $n^{\circ}$ 1.544. Informe de POCASA-Porriñesa de Canteiras, S.A. (inédito). Tomo II: Memoria, 146 pp., y Planos, $34 \mathrm{pp}$

Zadeh, L.A., 1965. Fuzzy sets. Information and Control 8, 338-353.

Recibido: diciembre 2015

Revisado: febrero 2016

Aceptado: abril 2016

Publicado: junio 2017 